\title{
Transatlantica
}

Revue d'études américaines. American Studies Journal

\section{(R)évolution américaine : Les peintres de la jeune République}

A propos de l'exposition de la Corcoran Gallery, The American Evolution : A History through Art, $1^{\mathrm{er}}$ mars - 27 juillet 2008, Washington D.C.

\section{François Dambre}

\section{(2) OpenEdition}

1 Journals

\section{Édition électronique}

URL : https://journals.openedition.org/transatlantica/4319

DOI : $10.4000 /$ transatlantica.4319

ISSN : $1765-2766$

Éditeur

Association française d'Etudes Américaines (AFEA)

\section{Référence électronique}

François Dambre, «(R)évolution américaine : Les peintres de la jeune République », Transatlantica [En ligne], 1 | 2009, mis en ligne le 02 septembre 2009, consulté le 16 septembre 2021. URL : http:// journals.openedition.org/transatlantica/4319; DOI : https://doi.org/10.4000/transatlantica.4319

Ce document a été généré automatiquement le 16 septembre 2021.

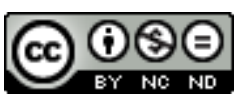

Transatlantica - Revue d'études américaines est mise à disposition selon les termes de la licence Creative Commons Attribution - Pas d'Utilisation Commerciale - Pas de Modification 4.0 International 


\section{(R)évolution américaine : Les peintres de la jeune République}

A propos de l'exposition de la Corcoran Gallery, The American Evolution : A History through Art, $1^{\mathrm{er}}$ mars - 27 juillet 2008, Washington D.C.

\section{François Dambre}

1 Qu'y a-t-il de commun entre le portrait compassé de George Washington par Gilbert Stuart (George Washington, 1803) et l'image pieuse d'un Mao Zedong revue et corrigée par Andy Warhol près de deux siècles plus tard (Mao, 1973)? Telle était la question liminaire que posait l'exposition de la Corcoran Gallery, The American Evolution: a History through Art. Délaissant la chronologie au profit d'une approche thématique et comparative, The American Evolution rassemblait près de 200 peintures, photographies et sculptures autour de cinq axes présentés comme fondateurs de l'identité nationale américaine : l'argent, la terre, la politique, l'échange culturel et le monde moderne. On s'intéressera ici en particulier aux artistes de la jeune République présents dans l'exposition'1.

Les premières salles, placées sous le signe de l'argent, donnaient à voir le commerce des esprits comme celui des marchandises.

3 Joseph Blackburn (Portrait of a Gentleman, 1760) et John Singleton Copley, portraitiste à succès des commerçants de Boston (Thomas Amory II, 1770-1772) y côtoyaient Charles Bird King (Poor Artist's Cupboard, 1815), pour lequel la jeune République, loin d'être une nouvelle Rome républicaine, semble une province hostile au développement des beaux arts. Le tableau de Bird King montre ainsi les maigres effets personnels d'un artiste en quête de reconnaissance sociale et artistique. Il y est fait mention ironique, sur un parchemin, de preuves selon lesquelles Philadelphie serait pourtant «the Most Hospitable, the Greatest Patron to the Fine Arts, and in Every respect Superior to any City in the World ${ }^{2}$ ». Dans la jeune République, le soutien à la création est en effet fort limité, hormis les commandes pour les édifices publics. L'autonomisation esthétique des acteurs du champ artistique vis-à-vis de l'ancienne puissance coloniale est 
graduelle. C'est en 1825, à New York, nouvelle capitale des arts américains, qu'est inaugurée la National Academy of Design.

4 Avec l'essor du commerce maritime et de l'industrie émerge une élite qui aime se dépeindre dans des intérieurs opulents. Gilbert Stuart (Edward Shippen, 1796), James Peale (Marcia Burns [Mrs. John F. Van Ness], 1797), Matthew Pratt, Charles Peale Polk (Thomas Corcoran, 1802-1810), ou encore Joshua Johnson (Grace Allison McCurdy [Mrs. Hugh McCurdy] and Her Daughters, Mary Jane and Letitia Grace, 1804) sont les élégants portraitistes de cette société patricienne ${ }^{3}$. Originaire du Maryland, Joshua Johnson ${ }^{4}$ est l'un des premiers peintres africains-américains des Etats-Unis. Redécouverte en 1939, son œuvre pose la question de la place marginale de la représentation des AfricainsAméricains dans la production picturale, de source eurocentrique, de la jeune nation. Kerry James Marshall, présent dans les salles de l'exposition consacrées à la politique, s'interroge avec force dans Voyager (1992) sur les traces de la mémoire dans la diaspora noire.

5 A nouvelle histoire, nouveaux lieux de commémoration et nouveaux rites républicains. Mount Vernon devient un lieu de pèlerinage laïc avec Jennie Bellows Millard (Mount Vernon, 1850) tandis que se multiplient les sculptures (Jean-Antoine Houdon: Mask of George Washington, 1785) et les portraits flamboyants du héros de Yorktown. Les artistes patriotes John Trumbull et Gilbert Stuart réalisent maints portraits de «Son Excellence ", fabriquant ainsi toile après toile une iconographie républicaine. La jeune République se crée une mythologie autour d'événements fondateurs (Rembrandt Peale : Washington Before Yorktown, 1824) comme autour d'une exaltation du quotidien (Frank Blackwell Mayer : Leisure and Labor, 1858). En 1822-23, Samuel F. B. Morse ${ }^{5}$, plus connu pour ses innovations techniques que pour son entreprise picturale, peint avec une précision quasi scientifique les différents acteurs de la vie politique (The House of Representatives). Dans la lignée de peintres d'histoire comme West et Trumbull, la toile de Morse porte sur l'ultra-contemporain en montrant avec fierté le fonctionnement régulier du nouvel ordre démocratique.

$6 \mathrm{Au}$ fur et à mesure que se construit la nation américaine, c'est une autre vision du territoire qui se donne à lire. Le paysage est considéré comme un livre vierge et infini où peut s'écrire l'histoire spectaculaire d'un destin national, manifestation d'une volonté divine. Les peintres délaissent la représentation topographique (Thomas Birch : View of the Delaware near Philadelphia, 1831) ou les scènes de genre (George Caleb Bingham : Cottage Scenery, 1845) au profit d'une évocation spectaculaire d'un paysage original perdu avant même d'avoir été complètement appréhendé6.

7 La nature primitive, reflet du dessein divin, inspire les artistes influencés par les écrits d'Alexander von Humboldt. Frederic Edwin Church peint en 1857 Niagara, œuvre aux dimensions monumentales $(107,3 \times 230 \mathrm{~cm})$, qui répond aux préoccupations d'une Amérique cherchant une vision panoramique de sa destinée. Les chutes du Niagara représentent une source de profits pour le peintre, qui perçoit un droit d'entrée des spectateurs voulant un contact avec une nature perçue comme sauvage et indomptable.

Lancée en Ecosse par Robert Barker en 1788, la peinture panoramique a connu un rapide développement aux Etats-Unis. En 1801-03, John Vanderlyn peint, à l'instigation d'Aaron Burr, une série de vues des Chutes qu'il commercialise ensuite (sans beaucoup de succès) sous forme de gravures. Vanderlyn construit en 1816 une véritable rotonde à New York afin d'y présenter ses vues grandioses du Château de Versailles (Panoramic View of the Palace and Gardens of Versailles, Metropolitan Museum of Art, New York). La 
vallée du Mississippi fait également l'objet d'immenses représentations panoramiques à partir des années 1840-50, avec des peintres comme John Banvard, John Rawson Smith, Henry Lewis, Léon Pomarède et Samuel B. Stockwell.

De Stuart à Warhol, The American Evolution met en lumière l'évolution des arts visuels depuis 1776 et rappelle que ces derniers ont toujours eu un rôle majeur dans le roman national d'une Amérique en constante redéfinition de son identité comme de son rapport à la représentation. Entre le portrait de George Washington en 1803 et celui de Mao en 1973, la peinture américaine s'est définitivement affranchie de ses racines figuratives européennes pour devenir jaillissante, immersive et gestuelle ${ }^{7}$. Les images parfois guindées-des origines et les symboles nationaux sont devenus dans l'Amérique de l'après-guerre un terrain de jeu et de détournement. Jasper Johns redessine la bannière étoilée (Flag, 1954-55, MoMA, New York) ou la carte de l'Amérique (Map, 1961, MoMA, New York). Sante Graziani re-visite la toile de Stuart en 1965 (Stuart's Red, White and Blue). L'atelier serein de Stuart a ainsi laissé la place à la frénétique Factory de Warhol, cette usine à fabriquer sans fin des images miroirs en contrepoint d'une culture de masse en quête d'éphémère et de sensationnel ${ }^{8}$.

De la révolution au concept d'évolution permanente, The American Evolution suggère que le projet politique américain, à l'instar de ses représentations picturales, se pense comme une œuvre ouverte, mouvante comme peut l'être aussi notre rapport aux images.

\section{NOTES}

1. The American Evolution a accueilli au total 19345 visiteurs. Il n'existe pas de catalogue consacré à l'exposition, mais une brochure et une sélection des œuvres sont disponibles sur le site internet de la Corcoran Gallery: http://www.corcoran.org/americanevolution/introduction.htm. Le catalogue des chefs d'œuvre de la Corcoran, publié sous la direction d'Eleanor Heartney, est également utile : A Capital Collection : Masterworks from the Corcoran Gallery of Art (Washington, D.C., Corcoran Gallery of Art, 2002).

La prochaine exposition de la Corcoran Gallery, de mars à juillet 2009, proposera une réflexion sur la question du paysage et de la représentation topographique, avec les sculptures et installations de Maya Lin, connue pour son intervention au Vietnam Veterans Memorial (Maya Lin : Systematic Landscapes).

2. Lecture détaillée du tableau effectuée le 15/07/2008.

3. Ils seront suivis par John Singer Sargent (Margaret Stuyvesant Rutherfurd White [Mrs. Henry White], 1883) ou William Merritt Chase (William Andrews Clark, 1915).

4. Sur Joshua Johnson, voir l'article de Jennifer Bryan et Robert Torchia: «The Mysterious Portraitist Joshua Johnson ", Archives of American Art, vol. 36, no. 2, 1996.

5. Sur Samuel F.B. Morse, voir l'article de François Brunet, « Kenneth Silverman. Lightning Man, The Accursed Life of Samuel F.B. Morse», Transatlantica, 2005, Couleurs d'Amérique, mis en ligne le 24 avril 2006, URL : http://transatlantica.revues.org/document873.html.

6. Partisans d'une esthétique du frisson, les peintres de la Hudson River School (bien représentée dans l'exposition) proposeront une vision spectaculaire des montagnes et des plaines de l'ouest : 
Worthington Whittredge (Trout Brook in the Catskills, 1875), Asher B. Durand (The Edge of the Forest, 1871) et Albert Bierstadt (Mount Corcoran, 1876-77).

7. L'exposition Pollock de novembre 1943 marque une véritable rupture qui va momentanément éclipser la production picturale européenne. C'est pourtant à cette même époque que naît par exemple en France un renouveau dans la peinture non-figurative, avec les peintres de l'abstraction figurative tel Alfred Manessier.

8. Retour provocateur (?) à la « vieille Europe », c'est dans les appartements royaux du Château de Versailles que les œuvres de l'artiste contemporain Jeff Koons sont actuellement exposées (jusqu'au 4 janvier 2009). Pour en savoir plus : http://www.jeffkoonsversailles.com.

INDEX

Thèmes : Trans'Arts

\section{AUTEUR}

\section{FRANÇOIS DAMBRE}

François Dambre : agrégé d'anglais, diplômé de l'Institut d'Etudes Politiques de Paris (1996). Prépare une thèse sur les arts visuels de la jeune République, sous la direction de Naomi Wulf (Paris 3). Enseigne l'anglais à l'Université des Antilles-Guyane (Martinique). 